\title{
Les sensations : Tout est une question de perspective
}

\author{
par Zack Dumont
}

$\mathrm{E}_{\mathrm{a}}^{\mathrm{n}}$ n tant que fonction physiologique, la vue n'a certainemenpas la vie facile. Habituellement, nous critiquons sa façon de s'imposer aux autres sens : nous fermons les yeux pour bien entendre, ressentir, goûter et sentir. Inversement, les autres sens semblent servir à la renforcer.

$\mathrm{Au}$ cours des derniers mois, la direction de la Société canadienne des pharmaciens d'hôpitaux (SCPH) a recueilli de l'information de diverses manières pour faire progresser la vision de notre Société (voir le récent commentaire présidentiel «Oser la différence "; Journal canadien de pharmacie hospitalière 2019;72[6]:469). Nous avons fureté partout et suivi des pistes prometteuses pour dénicher de belles occasions potentielles et nous préparer à affronter les menaces. Nous nous sommes délectés, nous avons absorbé les congrès de l'SCPH (savouré les calembours au sujet des excès alimentaires, qui ont égayé la conférence), dévoré le contenu du Journal et profité de tous les produits et services de la Société. Nous avons écouté, mené un sondage auprès des membres, organisé un sommet de planification stratégique inclusive et maintenant, nous menons une série de discussions ouvertes qui commencent à la Conférence sur la pratique professionnelle. Ces activités nous ont permis d'entendre les membres s'exprimer au sujet des problèmes qu'ils rencontrent dans le domaine de la pharmacie. Et pour finir, je demande la permission d'étendre l'analogie pour la bonne cause : l'utilisation $\mathrm{du}$ « toucher " pour symboliser les soins; nous sommes restés bien conscients des personnes pour qui nous entreprenons tout cela : les patients.

À mesure qu'elle s'approche du lancement du plan stratégique, la SCPH subit un certain nombre de changements. Nous évaluons tous les processus pour être certains de faire les choses pour les bonnes raisons et non pas " parce qu'elles ont toujours été faites ainsi ». Des solutions technologiques actuelles et conviviales visant à mieux interconnecter les membres (p. ex. les réseaux des spécialités en pharmacie) et les parties prenantes (p. ex. un nouveau site Web) sont à l'étude. Des options mieux centrées sur les membres remplacent les anciens services. De plus, nos comités ont fait l'objet d'un examen et d'un réalignement pour que les bénévoles consacrent leur temps et leurs efforts à la stratégie, tandis que le bureau prend les rênes des opérations. Bien que l'aiguillon du changement puisse piquer, ces actions visent à « combler les sens » le moment venu.

À ce propos, notre vision ne peut rester fixée uniquement sur le court terme. Nous positionnons la Société en vue d'une croissance importante au cours des années à venir, de manière à bénéficier d'une communauté avec laquelle il est possible d'aborder les problèmes de la pharmacie hospitalière à très long terme. Cela dit, quelle est votre vision? Non seulement de votre Société, mais également de votre profession de pharmaciens? Dans quelle mesure êtes-vous sûrs qu'elle est partagée par votre voisin œuvrant dans l'autre hôpital ou qu'elle s'est imposée dans la région ou la province voisine? Dans quelle mesure êtes-vous certains de prendre en compte tous les points de vue? La vision claire de la pharmacie hospitalière canadienne dépend de l'intégration d'autant de points de vue que possible. Des cliniques de première ligne aux hôpitaux - les grands et les petits; les femmes, les hommes et les autres; les personnes nées au Canada et les nouveaux arrivants; les jeunes professionnels et les plus expérimentés. Plus la contribution sera diversifiée et inclusive, plus la vision sera incontestable. C'est maintenant le moment où jamais d'entendre nos parties prenantes au complet. Nous devons écouter l'avis de tous nos membres actuels et en accueillir davantage. Nous devons favoriser un environnement sécuritaire qui encourage les membres à s'exprimer. Et nous devrions disposer de la meilleure unité de traitement prête à recevoir ces messages et à les transformer en quelque chose de plus grand. L'un de nos nouveaux comités - Vision de la pratique pharmaceutique - a été constitué pour être le catalyseur de ce processus. Restez à l'affût des occasions favorables qui contribuent à façonner notre profession.

Préparez-vous à vous concentrer sur l'essentiel au cours de ces prochaines années, et nous nous efforcerons ensemble de réaliser cette vision de la Société et de l'ensemble de la pharmacie hospitalière. Tant que nous utilisons tous nos sens pour rester ouverts à une perspective collective, la vue - la vision - peut être « sens-ationnelle».

[Traduction par l'éditeur]

Zack Dumont, BSP, ACPR, M. S. (Pharm.), est président élu et liaison interne de la Société canadienne des pharmaciens d'hôpitaux. 